Document downloaded from:

http://hdl.handle.net/10251/81426

This paper must be cited as:

Conejero, JA.; Jordan-Lluch, C.; Sanabria-Codesal, E. (2016). An algorithm for selforganization of driverless vehicles of a car-rental service. Nonlinear Dynamics. 84(1):107114. doi:10.1007/s11071-015-2237-4.

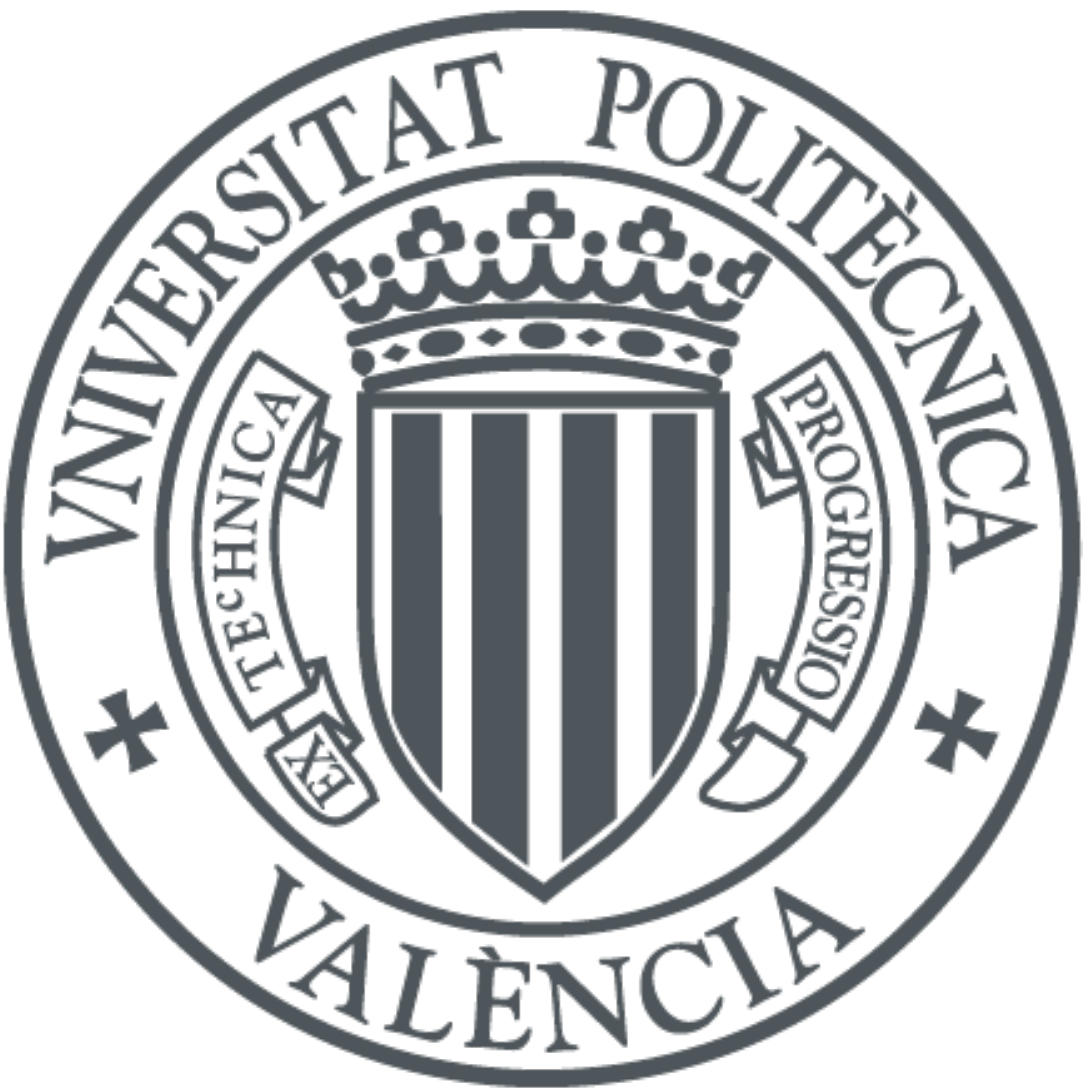

The final publication is available at

DOI $10.1007 / \mathrm{s} 11071-015-2237-4$

Copyright Springer Verlag (Germany)

Additional Information

The final publication is available at Springer via http://dx.doi.org/10.1007/s11071-015-22374 


\title{
An algorithm for self-organization of driverless vehicles of a car-rental service
}

\author{
J. Alberto Conejero • Cristina Jordán • \\ Esther Sanabria-Codesal
}

Received: date / Accepted: date

\begin{abstract}
The solution of fleet assignment problems is one of the core points in the management of a car-rental company. These problems arise from the restrictions imposed by the acceptance of reservations of the customers and from the necessity of an arrangement of the cars at the different depots for attending these bookings. However, with the coming of driverless cars the rearrangement of vehicles among the depots can be simplified since no staff must be responsible of these tasks. In this note we show a heuristic algorithm that permits to minimize the number of cars that have to be subcontracted from an external provider in order to attend a list of reservations from customers. Its efficacy is shown in comparison to the solution given by the Integer Linear Programming method. These results can be of interest for other autonomous systems that need to be controlled.
\end{abstract}

Keywords fleet assignment problem · car-rental services · integer linear programming $\cdot$ heuristics $\cdot$ graph-theory $\cdot$ time-space networks

Mathematics Subject Classification (2000) 05C21 68R10 $90 \mathrm{~B} 35$

\section{Introduction}

The management of a car-rental company presents an interesting problem from the point of view of operations research, because it can comprise the logistic process

J. Alberto Conejero

Instituto Universitario de Matemática Pura y Aplicada (IUMPA)

Universitat Politècnica de València, E-46022 València, Spain.

E-mail: aconejero@upv.es

Cristina Jordán

Instituto Universitario de Matemática Multidisciplinar (IMM)

Universitat Politècnica de València, E-46022 València, Spain.

E-mail: cjordan@mat.upv.es

Esther Sanabria-Codesal

Departamento de Matemática Aplicada (DMA)

Universitat Politècnica de València, E-46022 València, Spain.

E-mail: esanabri@mat.upv.es 
starting from the transportation and deployment of cars to the company depots until the very moment that a car is picked up by the client [?]. This includes the revenue management for finding the optimal equilibrium between the price offered to the clients and the utilization of the vehicles, the optimization of the solution to the fleet assignment problem of locating the cars in order to attend the bookings, and the control of the inventory trying to attend the demand forecasts. We refer to [?] and the references inside for an overview on the mathematical problems that arise in the car-rental industry.

When studying the operational costs, the whole process starting from the car manufacturer, considering the fleet movements, reservations and transfers, and finishing with the sale of the car to a reseller is considered [?]. See also the challenge found in the 1999 edition of the ROADEF Challenge [?] and the improvements respect to those results presented in [?].

For minimizing these costs, a model based on a specific minimum cost timespace network is usually considered, see $[?, ?, ?]$. In these networks, a large number of nodes is considered for representing the different locations at different times. Then, the problem can be seen as an Integer Linear Programming (ILP) model, where the objective function is designed in order to maximize the benefit, minimize the cost, or to optimize the use of the vehicles. This has been in part the basis of a number of optimization algorithms in the airline industry, e.g. [?,?,?,?], and in the train industry, e.g. [?,?], and for finding possible optimal locations for facilities in an urban setting [?].

A huge complication in the management comes from the acceptance of one-way reservations since they can produce an accumulation of vehicles at certain depots. Then some of the vehicles are relocated by means of trucks or other freight manner. Nevertheless, these reservations are usually permitted because the quality of the caring service is one of the cornerstones for the success of a company in the carrental industry. To compensate the costs in which the company incurs, a penalty fare is usually applied to these clients.

In a short future, the transport policy in many European countries is to increase the share of sustainable travel modes. This is one of the pillars of the transition of our cities to the smart city concept. Some pilot experiences have been developed under the FP7 and H2020 programs of the European Union, see for instance [?,?]. An example of implementation of these practices in the city of Helsinki can be found in [?]. On the other hand, start-up's like RelayRides [?] try to take profit of the new sharing economy applied to the renting of cars by particulars.

During the 2000's, great advances in robotics and artificial intelligence have permitted to develop prototypes of autonomous cars. The advances can be followed looking at the DARPA Grand Challenge competition [?]. After the last news on Google driverless cars [?], motor companies like Audi or BMW have presented their first models very recently [?]. Thus, with the progressive introduction of driverless cars, it is expected that in urban cities most of the population will lack of owned cars and will take either public or shared transports.

In this last case, the use of shared or rented cars will considerably increase. The use of driverless cars will allow a reduction of the operational costs needed for rearranging the cars at the depots.

In this note we consider the logistics of a car-rental company of driverless cars with several depots and an initial number of cars at each depot. If required, the cars can move on their own from a depot to another depot. Taking this into account, 
we can study the problem of how to organize the company fleet of vehicles for attending a list of reservations given in advance. The problem will be tied to the following considerations:

(C1) A number of reservations is given. We consider that the satisfaction of all customer petitions is mandatory. So that, all reservations must be accepted.

(C2) If some cars are needed at a depot at the initial time, then we bring there from an external provider.

(C3) If there are not enough cars available at a depot $i_{0}$ at some time $t_{0}$ (not the initial), then we first try to bring cars from another depot $i_{0}^{\prime}$. This is only done if we do not leave unattended bookings at this depot before time $t_{0}$.

(C4) If after these rearrangement of vehicles, there are not enough cars available at any depot to attend a booking, then we subcontract them from an external provider.

This problem can be compared with the aforementioned ones in [?,?] and with the one of the management of an electric car-rental service [?].

In this paper we present an heuristic algorithm that permits to obtain an optimal solution to the problem in terms of reducing the need of cars from an external provider. Its efficiency is compared with the solution obtained by solving the corresponding integer linear programming model.

The paper is organized as follows: First, in Section 2 we set our model that is based on a time-space network. In Section 3 we pose this problem as an optimization problem to be solved using an (ILP) model. The heuristic algorithm proposed for solving the aforementioned problem is explained in Section 4. In Section 5 we discuss the results obtained by both procedures. Finally, in Section 6 we briefly report the conclusions.

\section{Description of the model for managing a list of reservations}

The model for representing the logistics of the car-rental service will be a timespace network. A directed network $N=(V, E)$ is given by a set of nodes $V$, and a set of ordered pairs of nodes (arcs). If an arc connects the node $u$ with the node $v$, we will simply denote it by $(u, v)$. Given a network $N$, we can consider flows on them. A flow $f$ is a function $f: E \rightarrow \mathbb{N}$. For every node $u \in V$ with positive incoming and outgoing degree, we have that the conservation law of the flow holds:

$$
\sum_{v \in V,(v, u) \in E} f(v, u)=\sum_{v \in V,(u, v) \in E} f(u, v) .
$$

In particular, we will consider time-expanded networks, also called time-space networks [?,?]. In these networks, the inner nodes of the network (not the sink nor the source) represent locations at certain times.

Consider that a car-rental service is operating in a city with $n$ locations (depots) and assume that depot $i$ starts with a given number of cars $k_{i}$. Let $k_{0}$ be the total number of cars of the company, that is $k_{0}=\sum_{i=1}^{n} k_{i}$. We also consider that we have received reservations within $m$ consecutive times represented by the sequence $\left\{t_{j}: 1 \leq j \leq m\right\}$. Adicionally, we have considered $t_{m+1}$ for counting how many cars we have at the parking of each depot. Then our network $N=(V, E)$ will have a set of nodes $V$ that consists of a source node $S$, a node $P$ for representing 